\section{Remarkable and novel increase}

SIR - There has always been a striking difference between scientists and physicians given to flamboyant accounts of their own work and those who prefer a more modest account or telling understatment. With similar pieces of work, one group might go into print with a paper on "Intrinsic DNA polymerase activity of vegetable material extracted from Hevea brasiliensis", while another group would startle us all with "New life out of old rubber tyres".

Prompted by such considerations, I have recently scanned the biological literature (listed in Index Medicus and Medline) for evidence of changing trends in the selection of evocative words used in the titles of papers. A 30-year period from 1966 to 1995 was considered in five-year blocks. The period 1991-95 has been calculated on the basis of the rates from January 1991 to August 1994, and then extrapolated to December 1995, assuming no change in the trends over that period.

The total number of publications between 1966 and 1970 was 986,670 . This has increased in a more-or-less linear manner to a predicted total of $1,801,430$ for the years 1991-95. During this period, the number of papers with the words novel, new, exciting or remarkable in the title increased also in a linear manner from $19,857(1966-70)$ to 45,521 (1991-95). Other words, such as reliable, secure, consistent or precise might be considered equally evocative, although rather less strident. These words were used relatively infrequently, but they increased nevertheless from 204 (1966-70) to 1,004 (199195 ). It will be apparent that the use of all 8 words has increased more rapidly than the total number of papers over the same period. Furthermore, the increase in the use of type 2 words (reliable, secure, consistent or precise) has outstripped the increase in the use of type 1 words (novel, new, exciting or remarkable). There is an exponential decrease in the ratio of the use of type 1 words to type 2 words during the interval 1966-95, which requires some explanation.

There seems to be a general trend in the scientific community (among whom none is more guilty than the present correspondent) to elaborate the titles of scientific publications by the use of evocative words and phrases. Possibly the increased use of reliable, secure, consistent or precise reflects a willingness to concede to the anticipated preference of referees, who might be thought too wise to fall for such obvious self-aggrandisement as that

Letters submitted for Correpsondence should be typed, double-spaced, on one side of the paper only. generated in an author by the use of novel, new, exciting or remarkable. However, at a time when the recruitment of the most able school-leavers into science is increasingly threatened, we should not perhaps go too far down the road of self-effacement, but try rather to convey the excitement of discovering that you really can make new life out of old rubber tyres.

\section{John MacDermot}

Department of Clinical Pharmacology,

Royal Postgraduate Medical School,

Du Cane Road,

London W12 ONN, UK

\section{Alas, poor title}

SIR - A systematic computer search of recently published papers in the biomedical sciences has confirmed what our everyday experience had led us to suspect: that authors and editors of review papers (such as appear in the News and Views section of this journal) are predisposed to use one of three catch-phrases in making their titles. In the past five years, the phrase " $\mathrm{A}$ tale of two. .." has found its way into no fewer than 61 titles. Variations of "Of mice and men" have been used 32 times, and "Much ado about. .." a respectable 22 times. (Eight per cent of the offending titles were in Nature.) We call for an immediate moratorium on the use of these hackneyed titles. Surely other works of English literature with titles amenable to such pillage can be found.

If something is not done soon, we fear this trend will reach its logical conclusion with "Much ado about a tale of two mice and men".

\section{Anne Moon}

Nathan Machin

Department of Molecular and Cell Biology, 455 Life Sciences Addition,

University of California,

Berkeley, California 94720, USA

\section{Bright spark?}

SIR - Max Delbrück, were he alive today, would be amused to find himself described as an electrical engineer ("Gaps in molecular biology" Nature 372, 33; 1994). He initially studied astronomy, and completed a doctorate in theoretical physics, with Max Born as major professor (E. P. Fischer \& C. Lipson, Thinking About Science, Max Delbrück and the Origins of Molecular Biology; Norton \& Co., New York and London, 1988).

\section{Thomas C. Nelson}

500 West Hampton Avenue,

Milwaukee, Wisconsin,

USA

\section{Randomization}

SIR - In the Commentary article "Making medicine more scientific", Hiatt and Goldman suggest that "new statistical approaches" might somehow be able to rescue from computerized hospital records reliable information as to which treatment strategies produce the best long-term outcome for the patient ${ }^{1}$. If true, this would be wonderful: after all, a computerized analysis of data that already exist would be much easier than a randomized trial.

But it is not true. The reason is that where there is important uncertainty as to which of two treatment strategies to prefer, the real difference in long-term outcome might well be negligibly small, or it might well be of moderate (but humanly worthwhile) size, but it is unlikely to be large. So clinical research needs to be able to discriminate reliably between zero effects and moderate effects, and it cannot do this if it adopts methods that are potentially subject to moderate bias, or to moderate random error.

Clinical research must therefore use methods that guarantee that any biases will be negligibly small (which, in practice, can usually be achieved only by proper randomization, followed by appropriate statistical analysis), and that guarantee that the play of chance will likewise be small (which usually requires large numbers of patients to have been studied). Non-randomized methods, such as those now being introduced at great expense in the United States, cannot exclude moderate biases and so cannot yield reliable comparisons between plausible alternative treatment strategies.

Of course, randomization is not needed to show that prolonged cigarette use is a cause of most deaths from squamous cell lung cancer, for the excess among habitual cigarette smokers is so extreme ${ }^{2}$. But large-scale randomized evidence ${ }^{3}$ is certainly needed to determine whether or not today's chemotherapy can cure some of these patients, for the proportion cured is not large. If Hiatt and Goldman want medical education to help make medicine more scientific, then they should not mislead themselves or their students into supposing that "results approximating those from trials" can be "extracted from routine clinical data".

\section{Richard Peto}

Rory Collins

University of Oxford,

Clinical Trial Service Unit,

Nuffield Department of Clinical Medicine, Radcliffe Infirmary,

Oxford OX2 6HE, UK

1. Hiatt, H. \& Goldman, L. Nature 371, 100 (1994).

2. Peto R et al Mortality from Smoking in Developed Countries 1950-2000 (Oxford University Press, Oxford, 1994)

3. Peto, R., Collins, R., Gray, R. Ann. N.Y. Acad. Sci. 703 314-340 (1993) 